\title{
R\&D for the Vertexing at CLIC
}

\author{
Sophie Redford* \\ On behalf of the CLICdp collaboration \\ CERN \\ E-mail: sophie.redfordecern.ch
}

The Compact Linear Collider is a candidate to be the next high-energy particle physics collider. Using a novel acceleration technique, electrons and positrons would be brought into collision with a centre-of-mass energy of up to $3 \mathrm{TeV}$. Despite challenging levels of beam-induced background, this would provide a relatively clean environment in which to perform precision physics measurements. The vertex detector would be crucial in achieving this, and would need to provide accurate particle tracking information to facilitate secondary vertex reconstruction and jet flavour-tagging. With this goal in mind, current technological limits are being stretched to design a low occupancy, low mass and low-power dissipation vertex detector for CLIC. A concept comprising thin hybrid pixel detectors coupled to high-performance readout ASICs, power-pulsing and air-flow cooling is under development. In this paper, the CLIC vertex detector requirements are reviewed and the current status of R\&D on sensors, readout, powering, cooling and supports is presented.

The 23rd International Workshop on Vertex Detectors

15-19 September 2014

Macha Lake, The Czech Republic

${ }^{*}$ Speaker. 


\section{Introduction}

The Compact Linear Collider (CLIC) is a possible future multi-TeV electron-positron collider, which would use a novel two-beam acceleration scheme to achieve accelerating gradients of $100 \mathrm{MV} \mathrm{m}^{-1}$ [1]. After a staged construction [2], the maximum centre-of-mass energy $(\sqrt{s})$ would be $3 \mathrm{TeV}$. This would require a tunnel of almost $50 \mathrm{~km}$ in length. A high instantaneous luminosity, up to $6 \times 10^{34} \mathrm{~cm}^{-2} \mathrm{~s}^{-1}$ at $\sqrt{\mathrm{s}}=3 \mathrm{TeV}$, can be achieved by restricting the transverse size of the beams to $\sigma_{(x, y)}=(40 \mathrm{~nm}, 1 \mathrm{~nm})$. The beams are composed of dense bunch trains separated by $20 \mathrm{~ms}$ gaps, where each train consists of 312 bunches separated by $0.5 \mathrm{~ns}$ gaps. This low duty-cycle facilitates a trigger-less readout, power-pulsing and air-flow cooling of the vertex detector.

The small bunch sizes necessary for the desired luminosity also create high levels of beaminduced background. Photons radiated from the colliding bunches can interact, producing hadrons or electron-positron pairs. These background particles typically have low transverse momentum, and peak in the forward regions. However, their cumulative effect throughout the bunch train is significant. They must be removed from the physics event of interest (typically one per bunch train) by using time and momentum information.

If the beam-induced backgrounds can be controlled, a rich physics programme could be pursued at CLIC facilitated by the relatively clean environment of electron-positron collisions [3]. At the lowest energy stage, $\sqrt{s} \approx 350 \mathrm{GeV}$, a model-independent determination of the $\mathrm{ZH}$ coupling could be accessed through Higgsstrahlung and a top pair threshold scan could be performed. At the intermediate energy stage, $\sqrt{s} \approx 1.4 \mathrm{TeV}$, the top Yukawa coupling could be accessed through $\mathrm{t} \overline{\mathrm{t}} \mathrm{H}$ production, and precise measurements of many Higgs decay modes made. At the highest energy stage, $\sqrt{s}=3 \mathrm{TeV}$, the Higgs trilinear self-coupling and quartic HHWW coupling could be measured and many BSM models tested or explored.

\section{Vertex detector requirements}

To achieve this challenging physics programme, a detector capable of rejecting beam-induced backgrounds and performing precision measurements must be designed. Calorimeters with high granularity and ns-level timing are required to enable particle-flow reconstruction. An all-silicon tracker and instrumented steel return yoke will provide further tracking information. The $4-5 \mathrm{~T}$ solenoid field allows excellent momentum resolution at high energy, and contains most of the beam-induced background particles inside the beam pipe.

The vertex detector is of crucial importance in fulfilling the physics goals, as it will provide tracking information for displaced vertex reconstruction and jet flavour-tagging. A multi-layer barrel and end-cap system of hybrid pixel detectors is envisaged, around half a metre in length and with barrel radii from $30-70 \mathrm{~mm}$. Forward discs would provide coverage to low polar angles. The minimum inner radius is determined by the occupancy in the forward layers caused by the beaminduced backgrounds. At a radius of $30 \mathrm{~mm}$ from the interaction point, the maximum occupancy in these layers should be less than $3 \%$ (including a safety factor of 5 to account for uncertainty in the background simulation).

The vertex detector should provide a single-point resolution of $3 \mu \mathrm{m}$, which can be achieved with analogue readout and $25 \mu \mathrm{m}$ pixel size. To limit the amount of multiple scattering, a material 
budget of $0.2 \%$ of a radiation length per detection layer is imposed. This means there can be no active cooling elements, rather an air-flow system will remove heat from the vertex detector. For this to be feasible, the total power dissipated in the vertex detector region must not surpass $50 \mathrm{~mW} \mathrm{~cm}^{-2}$. Finally, hit time-slicing of $10 \mathrm{~ns}$ is required to reduce the level of beam-induced background affecting events of interest.

\section{Vertex detector layout}

The optimal positions of the sensor planes in the vertex detector have been studied in simulation to maximise the physics performance [4]. Two similar layouts were compared, using flavourtagging performance as a metric. Both layouts consisted of a barrel region and spiral end-caps to facilitate air-flow cooling (see Figure 1a). The first layout had 5 single-sided barrel layers and 4 single-sided end-cap layers. The second layout had 3 double-sided barrel layers and 3 double-sided end-cap layers. In both layouts, the layers were spaced equally between the same minimum and maximum dimensions, and the material of both layouts was approximately equal.

The position and number of layers in the vertex detector impact the number of hits made by a particle track, as shown in Figure 1a. However, the flavour-tagging performance of the two layouts for dijets at $200 \mathrm{GeV}$ was very similar, seen in Figure 1b. It was found that increasing the material by a factor of two in the double-sided layout had a much greater impact (Figure 1c). Double-sided layers will therefore be used in the vertex detector, as by sharing one thicker support the rigidity of two layers can be ensured without contributing twice to the material.

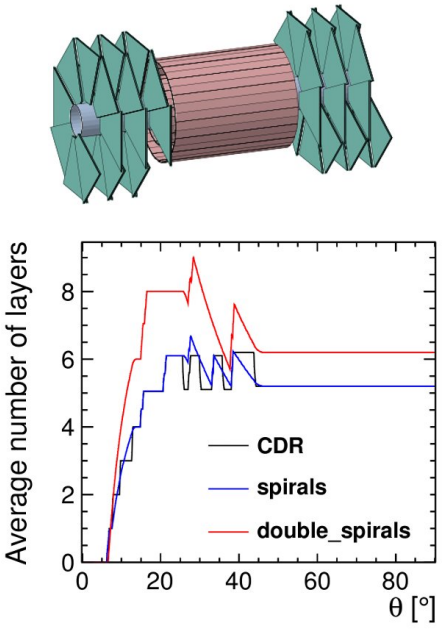

(a)

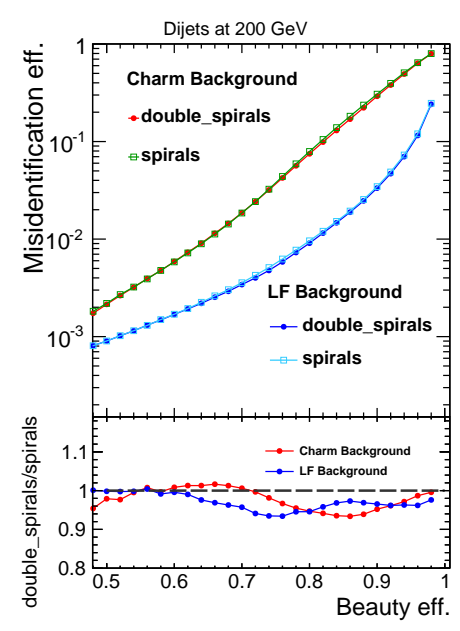

(b)

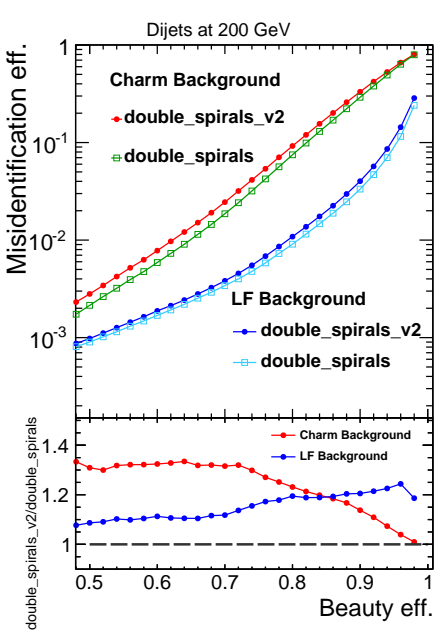

(c)

Figure 1: (a) top: the double-sided layout showing the spiral end-cap geometry. Bottom: number of layers as a function of the polar angle $\theta$ for the two layouts considered and the 'CDR' layout, consisting of singlesided layers and flat end-caps. (b): flavour-tagging performance for the two layouts considered. (c): flavourtagging performance for the double-sided layout compared with the same layout in which the material was increased by a factor of two (marked '_v2'). Taken from [4]. 


\section{Thin sensor assemblies}

The ultimate goal for the CLIC vertex detector is to use hybrid pixel technology consisting of a $50 \mu \mathrm{m}$ thick silicon sensor coupled to a $50 \mu \mathrm{m}$ thick readout ASIC via low-mass interconnects. Hardware R\&D into thin sensor assemblies is assessing the feasibility and performance of such devices. Timepix [5] and Timepix 3 [6] ASICs bump-bonded to sensors ranging from $50 \mu \mathrm{m}$ to $500 \mu \mathrm{m}$ thick have been characterised in lab tests and test beams. These ASICs have $55 \mu \mathrm{m}$ pixel size, considerably larger than the goal of $25 \mu \mathrm{m}$ pixel size for CLIC.

Sensor calibration was performed in lab tests at CERN and LNLS. Photons of known energy, either from radioactive sources or x-ray fluorescence, were used to measure the energy response of the Timepix in Time Over Threshold (TOT) mode. Using a variety of photon energies in the range of a few $\mathrm{keV}$ to $60 \mathrm{keV}$ allows a surrogate function to be fitted, which takes into account nonlinearities in the response. Once the calibration constants are know, TOT spectra can be converted into energy spectra, as shown in Figure 2.

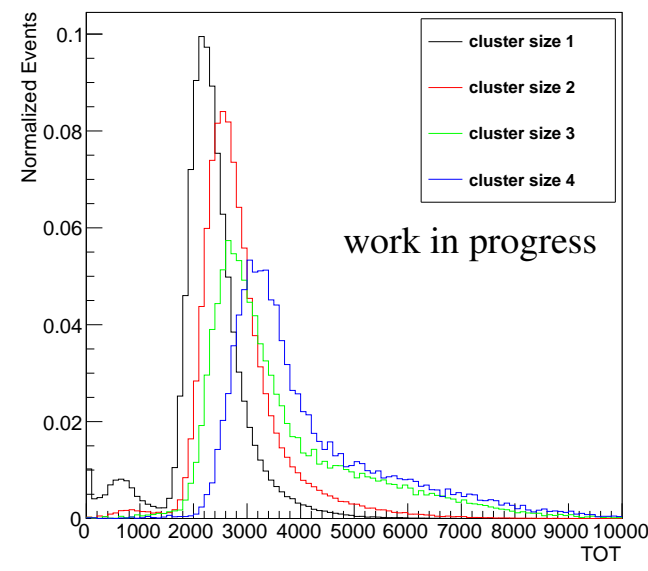

(a)

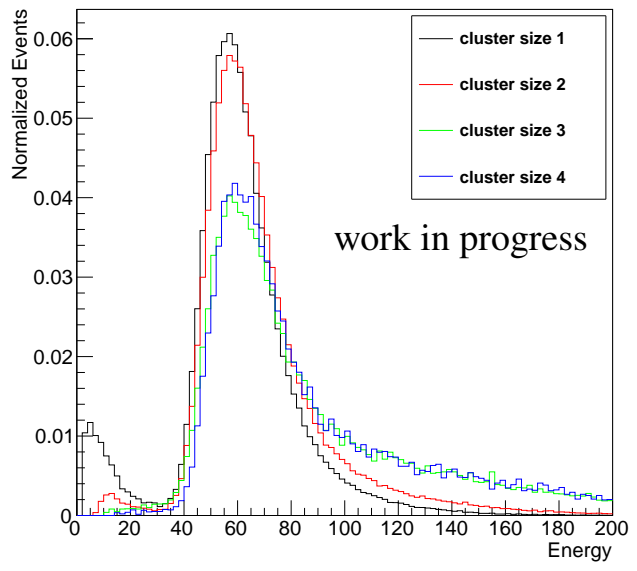

(b)

Figure 2: Uncalibrated (a) and calibrated (b) energy spectra of 1- to 4-hit clusters in a $200 \mu \mathrm{m}$ thick sensor. The units of energy are keV. Taken from [7].

Test beam measurements were performed on the assemblies at DESY and CERN. In both places, the EUDET telescope was used to provide track information with a position resolution at the Device Under Test (DUT) of $\sim 3 \mu \mathrm{m}$. The detection efficiency as a function of threshold was measured (see Figure 3), showing an efficiency above $99 \%$ at operating threshold for a $50 \mu \mathrm{m}$ thick sensor. The operating threshold is determined by taking $5 \sigma$ above the noise.

The overall resolution of an assembly is dependent on its thickness, as thicker sensors exhibit more charge sharing and the resolution of multi-hit clusters is better than that of single-hit clusters. Figure 4a shows the fraction of multi-hit clusters as a function of sensor thickness. For $50 \mu \mathrm{m}$ thick sensors, a single-hit cluster is produced $\sim 80 \%$ of the time. The resolution of a $50 \mu \mathrm{m}$ sensor is shown in Figure $4 \mathrm{~b}$. The two structures in this plot correspond to single-hit clusters, for which the 


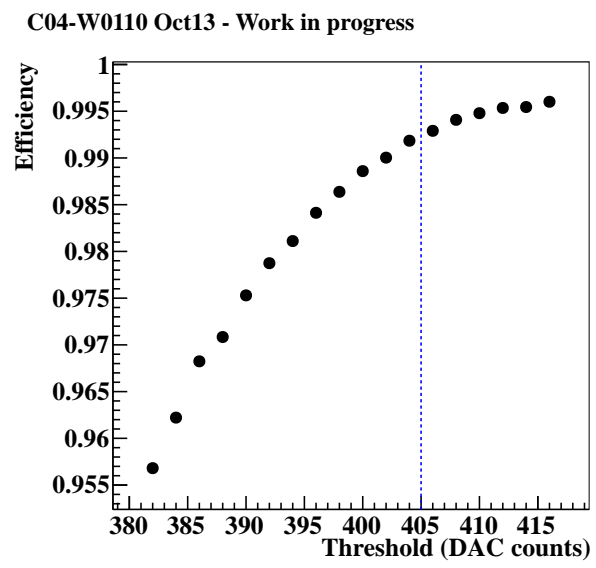

Figure 3: Efficiency as a function of threshold for a $50 \mu \mathrm{m}$ thick p-in-n sensor. The dashed line indicates the normal operating threshold for this assembly.

resolution is determined only by the pixel size, and two-hit clusters, which have a smaller resolution due to information from the relative charge in each pixel.

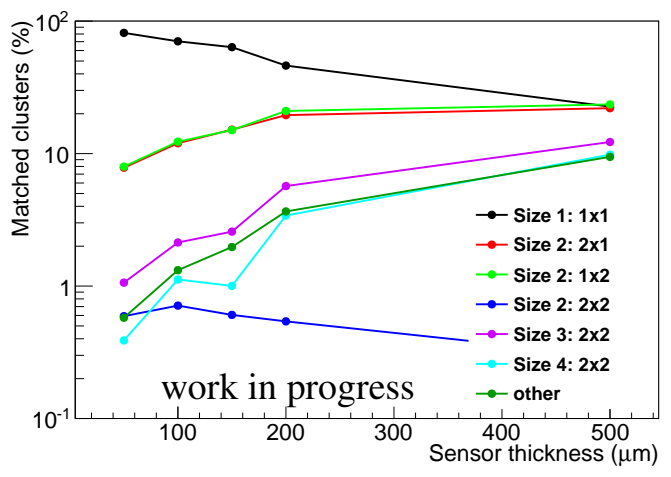

(a)

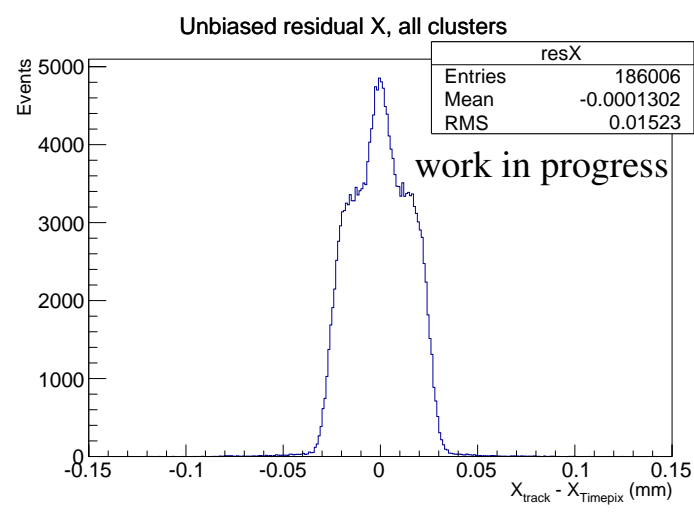

(b)

Figure 4: (a): the fraction of multi-hit clusters as a function of sensor thickness. 'Size' denotes the number of pixels in a cluster, ' $x \times y$ ' indicates the dimensions of the cluster. (b): the hit resolution of a $50 \mu \mathrm{m}$ thick sensor.

By calibrating the measured TOT values, the energy deposited in a sensor can be measured. Figure 5a shows the calibrated energy spectrum of a $200 \mu \mathrm{m}$ thick sensor, fitted with a Landau $\times$ Gauss convolution. The mean of the Landau indicates the most probable energy to be deposited in the sensor. By measuring this as a function of sensor bias voltage, the depletion voltage of the sensor can be determined, in addition to further characteristics such as dopant concentration and resistivity. The depletion voltage for this $200 \mu \mathrm{m}$ sensor is $\sim 30 \mathrm{~V}$, as shown in Figure $5 \mathrm{~b}$.

The CLICpix ASIC [8] has been designed to provide the necessary features for a readout chip at the CLIC vertex detector, including simultaneous time and energy measurements. It has $25 \mu \mathrm{m}$ pixel size, supports power-pulsing and is implemented in $65 \mathrm{~nm}$ technology. As a first step, this chip 


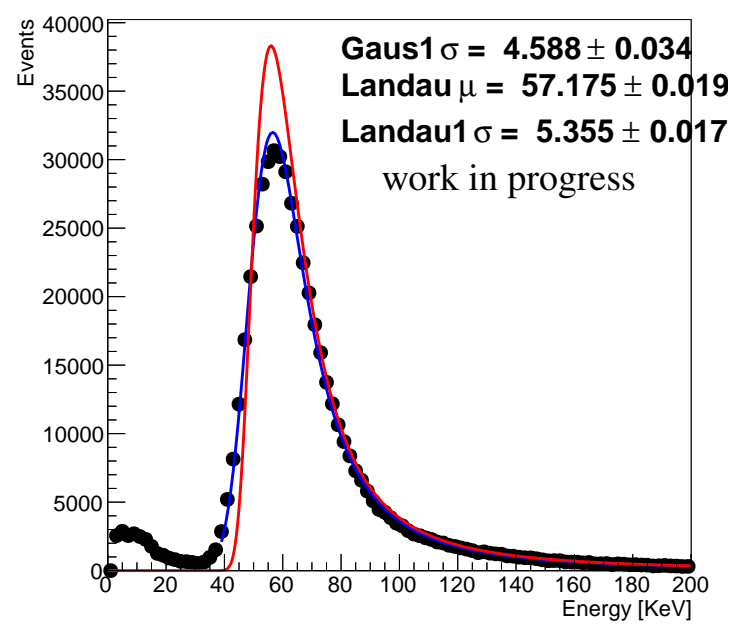

(a)

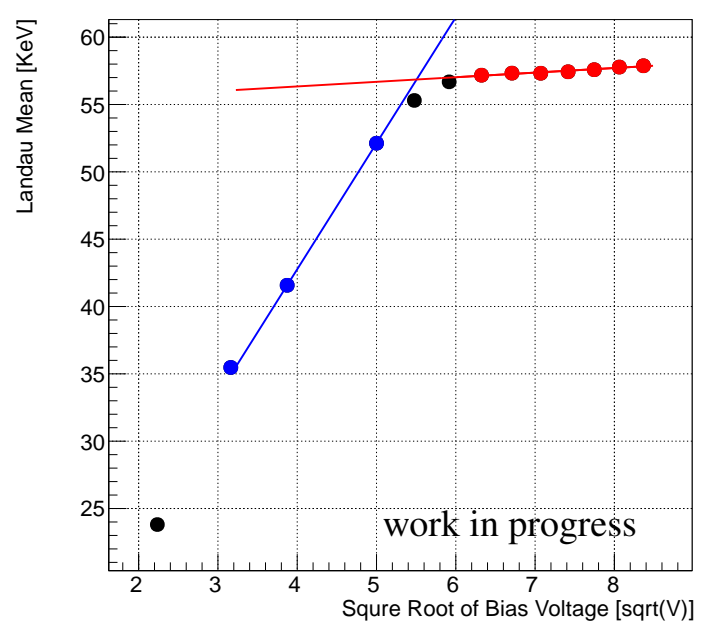

(b)

Figure 5: (a): calibrated energy spectrum from a $200 \mu \mathrm{m}$ thick sensor, fitted with a Landau $\times$ Gauss convolution (blue). The red line shows the Landau component. (b): most probable value as a function of bias voltage for a $200 \mu \mathrm{m}$ thick sensor. Straight lines are fitted to points of matching colour to determine the depletion voltage at their intersection. Taken from [7].

was glued to a CCPDv3 HV-CMOS sensor [9], creating a capacitive coupling between the two. The assembly was tested at the CERN PS test beam in August 2014 and successfully took data, showing a correlation between recorded hit position and reconstructed track position. Bump-bonding trials with planar sensors will test the feasibility of this operation with small pixel size.

\section{Power-pulsing and delivery}

Taking advantage of the low duty-cycle of the accelerator described in Section 1, the vertex detector will be power-pulsed in order to ensure that the heat dissipated in the vertex detector region remains below $50 \mathrm{~mW} \mathrm{~cm}^{-2}$. When the bunch trains collide in the detector, capacitors discharge to power the chips. Both analogue and digital domains in the chip will be on during collisions. During the $20 \mathrm{~ms}$ gap between bunch trains, the analogue electronics will be turned off. Digital electronics will be set to idle, except for readout. Data is read-off each of the 12 chips in a half-ladder in turn. This power-pulsing strategy reduces the power dissipated in the vertex detector region by several orders of magnitude, and has been demonstrated in the lab [10].

Power will be delivered to the CLICpix ASICs along thin aluminium flex cables by controlled current sources sitting outside of the vertex detector region. The ladders forming the barrel will each consist of 24 CLICpix assemblies, and will be powered from both ends in groups of 12 . Silicon capacitors and voltage regulators on each chip will provide local energy storage and voltage stabilisation. The material along the ladder necessary for this power delivery scheme has been estimated at $0.1 \%$ of a radiation length per layer. This is expected to drop by at least a factor of two, with anticipated improvements in storage capacitor power density and thinner conductors. 


\section{Air-flow cooling}

Despite minimising the power dissipated in the vertex detector, the total heat load remains $\sim 500 \mathrm{~W}$. To evacuate this heat, an air-flow cooling system is envisaged in which dry air at $0^{\circ} \mathrm{C}$ will be circulated through the vertex detector. The main advantage of this cooling system is the very low contribution it makes to the material budget. Simulations suggest that an air velocity of $\sim 5 \mathrm{~m} \mathrm{~s}^{-1}$ in the barrel should maintain temperatures below $40^{\circ} \mathrm{C}$ [11].

To better understand the mechanical challenges posed by the CLIC vertex detector, a thermomechanical test bench has been set up [12]. The test bench consists of a wind tunnel in which a stave is mounted on a turn-table and equipped with a heater and temperature sensors. The vibration of the stave in the air flow can be measured by an external laser.

The staves will be the supporting structures in the ladders of the vertex detector barrel. The material should contribute no more than $0.05 \%$ of a radiation length per layer. Current stave designs consist of thin carbon fibre layers sandwiching a foam or honeycomb core, with dimensions $1.8 \mathrm{~mm} \times 26 \mathrm{~mm} \times 280 \mathrm{~mm}$.

Figure 6 shows the RMS of the vertical displacement of the stave, recorded when the stave was at $90^{\circ}$ to the flow of air. The maximum vertical displacement of the stave for a $5 \mathrm{~m} \mathrm{~s}^{-1}$ wind speed was found to be $\sim 2.5 \mu \mathrm{m}$. This should not affect the single hit resolution of the vertex detector, as the displacement is in the same direction as the particle track. The power spectrum density of the stave was also measured, and the eigenmodes found. The frequencies identified are not thought to be problematic. The movement of the stave in the air flow has not yet been studied in simulation, however measured stave temperatures show good agreement with simulation for a range of wind speeds and power dissipation values.

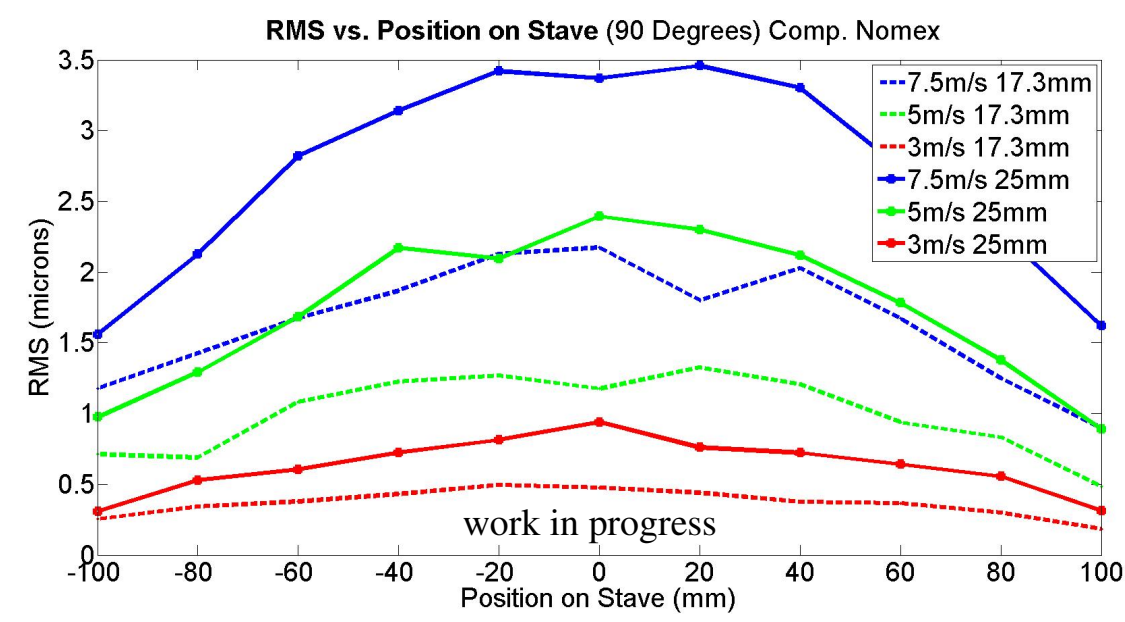

Figure 6: RMS of the vertical displacement of the stave as a function of position along the stave. The wind speed is indicated, as well as the channel depth. The air-flow was perpendicular to the stave.

A 1:1 scale model of the vertex detector is under preparation to increase the realism of these studies. This will mimic the injection of the air into the vertex detector and its flow through the spiral end-caps and around the barrel ladders. Cooling tests will be performed to further validate simulation. 


\section{Summary}

The CLIC accelerator and physics requirements make challenging demands of the vertex detector. Progress is being made in many areas to characterise and improve the performance of different components. R\&D is being carried out on thin sensors and readout chips, and a comprehensive test beam and lab measurement campaign has demonstrated that thin sensor assemblies with $55 \mu \mathrm{m}$ pixels can be operated with excellent precision and efficiency. The first assembly with $25 \mu \mathrm{m}$ pixels has successfully recorded hits, and further assessment of these small-pitch hybrid pixel detectors is planned. Powering, cooling and support strategies are being developed, with the objective of minimising the material of the detector. This can be achieved with power-pulsing and air-flow cooling. A single-stave test bench has been set up to verify thermo-mechanical requirements of the design, and a second more realistic multi-stave model is under development to further validate simulation.

\section{References}

[1] M. Aicheler, P. Burrows, M. Draper, T. Garvey, P. Lebrun, K. Peach, N. Phinney, H. Schmickler, D. Schulte and N. Toge (eds), A Multi-TeV Linear Collider Based on CLIC Technology: CLIC Conceptual Design Report, CERN-2012-007.

[2] P. Lebrun, L. Linssen, A. Lucaci-Timoce, D. Schulte, F. Simon, S. Stapnes, N. Toge, H. Weerts and J. Wells (eds), The CLIC Programme: Towards a Staged $e^{+} e^{-}$Linear Collider Exploring the Terascale: CLIC Conceptual Design Report, CERN-2012-005.

[3] L. Linssen, A. Miyamoto, M. Stanitzki and H. Weerts (eds), Physics and Detectors at CLIC: CLIC Conceptual Design Report, CERN-2012-003.

[4] N. Alipour Tehrani and P. Roloff, Optimisation Studies for the CLIC Vertex-Detector Geometry, CLICdp-Note-2014-002.

[5] X. Llopart, R. Ballabriga, M. Campbell, L. Tlustos and W. Wong, Timepix, a 65k programmable pixel readout chip for arrival time, energy and/or photon counting measurements, Nucl. Instr. and Meth. A $\mathbf{5 8 1} 485$ and erratum Nucl. Instr. and Meth. A 585106.

[6] T. Poikela et al., Timepix3: a 65K channel hybrid pixel readout chip with simultaneous ToA/ToT and sparse readout, JoI 9 (05) C05013.

[7] S. Maimon and S. Redford, Analysis of bias voltage scan data recorded with hybrid Timepix silicon pixel assemblies at the DESY testbeam, LCD-OPEN-2014-001.

[8] P. Valerio, R. Ballabriga and M. Campbell, Design of the $65 \mathrm{~nm}$ CLICpix demonstrator chip, LCD-Note-2012-018.

[9] I. Perić et al., High-voltage pixel detectors in commercial CMOS technologies for ATLAS, CLIC and Muзe experiments, Nucl. Instr. and Meth. A 731131.

[10] G. Blanchot, D. Dannheim and C. Fuentes, Power-pulsing schemes for vertex detectors at CLIC, CLICdp-Conf-2013-005.

[11] F. Duarte Ramos, H. Gerwig and M. Villarejo Bermudez, CLIC inner detectors cooling simulations, LCD-Note-2013-007.

[12] F. Duarte Ramos, Thermo-mechanical characterization of CLIC vertex detector ladders, LCD-OPEN-2013-004. 